\title{
Glosse
}

\section{Was ist gut für das Herz?}

_ Der Deutsche Herzkongress hat auch dieses Jahr eine Reihe neuer und aufregender Erkenntnisse präsentiert, die hier kurz zusammengefasst werden sollen:

- Die wirksamste primäre Präventionsmaßnahme bei kardialen Erkrankungen ist das gezielte Aussuchen der Eltern, da das Schicksal bzgl. KHK vor allem in den Genen liegt. Nur so kann letztlich der Durchbruch bei der Vermeidung kardiovaskulärer Ereignisse gelingen!

- Das LDL-Cholesterin sollte auf 0 oder sogar in den Minusbereich ge- drückt werden, egal wie; denn nur ein fehlendes LDL ist ein gutes LDL. Nach einer neuen Studie lebt man ohne LDL besser, wenn auch nicht länger.

- Beim Blutdruck hingegen sollte möglichst noch irgendein Wert messbar sein; sonst ist die Korrektur der übrigen Risikofaktoren nicht mehr sinnvoll.

- Kognitive Defizite schützen nicht vor Herzinfarkt, aber vor seinen Folgen, was die Lebensqualität betrifft. Dumme leben nach dem Infarkt fröhlich weiter, als sei nichts gewesen. Happy trotz broken heart!

- Menschen, die sich vermeintlich sehr gesund ernähren, also Vegetarier, werden nicht unbedingt älter, aber sie sehen meist so aus.

- Die Fortschritte in der Kardiologie sind so gewaltig, dass man sich seines eigenen Todes nicht mehr sicher sein kann. Die einzige Möglichkeit, der drohenden Unsterblichkeit zu entrinnen, ist die Non-Compliance.

- Dr. Peter Stiefelhagen

\section{Ein Prosit der Kardiologie}

\section{Bier bringt Wiesnherzen aus dem Rhythmus}

Von wegen Gemütlichkeit: Wer auf dem Münchner Oktoberfest zu oft den Maßkrug ansetzt, läuft Gefahr, Herzrhythmusstörungen zu entwickeln. Und die könnten ziemlich ungemütlich werden.

_ Herzen gibt es viele auf der Wiesn. Manche sind aus Lebkuchen und werden vor der Brust getragen. Die meisten aber klopfen in der Brust, und folgt man den Ergebnissen einer Münchner Studie, schlagen sie auf der Wiesn nicht nur höher, sondern auch schneller und unregelmäßiger. Schuld daran ist das ortsübliche Getränk.

Die arrhythmogene Potenz von Alkohol ist kein medizinisches Geheimnis. Die Zahlen, die Stefan Brunner und sein Team vorgelegt haben, sind dennoch ernüchternd. Hiernach weisen fast ein Drittel aller Insassen der alljährlich auf der Münchner Theresienwiese aufgestellten Bierbedürfnisanstalten elektrokardiografische Auffälligkeiten auf. Der Alkoholpegel muss dafür nicht einmal besonders hoch sein - doch je größer der Rausch, desto riskanter wird er fürs Herz.

Die Forscher hatten von rund 3.000 Wiesnbesuchern ein EKG per Smartphone aufgezeichnet und dabei auch den Alkoholpegel der Probanden bestimmt. Dieser lag im Mittel bei 0,86 mit einer Schwankungsbreite von 0 bis knapp 3 Promille. 30,5\% der Teilnehmer hatten einen gestörten Herzrhythmus. Am häufigsten waren Sinustachykardien (25,9\%). Es folgten Sinusarrhythmi- en und ventrikuläre Extrasystolen (jeweils $1,7 \%)$, Vorhofextrasystolen $(1,3 \%)$ und Vorhofflimmern oder -flattern (0,8\%). Den Berechnungen der Autoren zufolge steigt das Arrhythmierisiko mit jedem zusätzlichen Promille Blutalkohol um $75 \%$.

- Dr. Robert Bublak

- Brunner S et al. Eur Heart J 2017; online 26. April

\section{Das „Münchner Bierherz"}

Der Terminus "Münchner Bierherz" hat schon vor mehr als 100 Jahren seine Aufnahme in die medizinischen Fachbegriffe erzwungen. Neben den alkoholtoxischen Wirkungen sind beim Bierherz auch die großen Trinkmengen zu bedenken, die schließlich zur dilatativen Kardiomyopathie führen. Im damaligen Braugewerbe war es unter den Mitarbeitern nicht unüblich, täglich bis zu $15 \mathrm{Maß} \mathrm{Bier} \mathrm{zu} \mathrm{trin-}$ ken. Kaum auszudenken, was das Smart phone-EKG da angezeigt hätte. 